\title{
SCADA Backup System for the Control of Networked Valves in Modern Ships Facilities
}

\author{
E. Alexandropoulos \\ Dept. of Industrial Design and \\ Production Engineering \\ University of West Attica, Athens, \\ Greece
}

\author{
M. Papoutsidakis \\ Dept. of Industrial Design and \\ Production Engineering \\ University of West Attica, Athens, \\ Greece
}

\author{
N. Nikitakos \\ Dpt. of Shipping Trade \& \\ Transport \\ University of Aegean, \\ Greece
}

\begin{abstract}
Redundancy in critical control systems and ship's machinery in order to increase safety on ship, is necessary in modern ships. The project that was designed, implemented and is presented in this thesis, is trying to increase safety of a board, through the construction of a backup system that is able to control seawater valves. The installed SCADA system does not have a redundant control system for seawater valves which is a disadvantage. Control of the valves is achieved through the ship's installed SCADA system. The SCADA system consists of an HMI, the PLC in which the automation and control of the valves is executed, and the AS-i Bus (Actuator Sensor Interface). AS-i Bus (Network) consists of the AS-i Master (Gateways) that receives data from the PLC, through Profibus protocol and converts it to AS-i protocol in order to send data over AS-i cable to AS-i slaves. It also consists of AS-i cable that transfers data and power to the slaves. In order to create a backup system to control the seawater valves we have to program and install an HMI, a PLC and AS-i Master.The technology of the installed Control \& Monitoring System dates back to the first half of 2000. It is a question the compatibility of AS-i modules and PLC that are going to be used with the slave modules that have already been installed in the system.
\end{abstract}

\section{Keywords}

SCADA, PLC, AS-i Bus (Actuator Sensor Interface Bus), HMI, Valves

\section{INTRODUCTION}

The automization of industrial production as well as of shipping industry and ships has expanded, with the usage of SCADA systems and PLC given as a fact. The growth of the Information Technology has contributed a lot to the development that appears nowadays automation technology. The improvement of the material technology as well as the sensor technology, have also contributed to the development of automation technology. Modern ships are automated with a big and critical part of them being controlled by SCADA systems and PLC, through sensors and actuators being installed all over the ship. This allows the crew to control the ship from the Control Room, and have a good knowledge of performance and status of ship's machinery and systems.

Systems that are controlled by SCADA systems and PLC, are the most important for ship's operation. These are Propulsion Diesel Engines, Diesel Generators, Power distribution network, Air condition, Seawater cooling pumps and valves, freeing and compensating systems (including Valves, flowmeters and tank level). These systems are critical for the operation of a ship and many of them are redundant in order to achieve uninterrupted operation of the systems even if a malfunction occurs.
The present study has emerged from the necessity of existence of an alternative way to control the valves of a ship in order to control seawater networks on it. In this case, the control of the ship's valves and seawater networks is done through the installed SCADA system and PLC, either by the users or by programmed sequences. In case of a malfunction of the control system (HMI, PLC, etc.), there is no alternative way to control these valves remotely. This means that if the crew has to control the valves manually, then members of the crew must be moved locally the valves, with the assistance from the personnel in the control room, to control these valves manual, according to the instruction of the Chief engineer. To achieve the correct combination of the position of the valves (Open or Closed), in order to set the seawater network in the right way, many members of the crew must be used all over the ship, communicating with each other as well as with the control room. This is very difficult considering that the members of a crew in a ship are very few and that sometimes the weather is so heavy that the conditions in the ship are too difficult to deal with. Therefore, has been decided the design and implementation of a backup system, so as to be installed alongside with the already installed system, in order to control the seawater valves in case of malfunction of the installed control system. The system that control the ship's valves is part of the Control \& Monitoring system. The system that controls the seawater network and the valves on it, consists of an HMI (Human Machine Interface), a PLC (in which the automation program is executed), AS-i Network and ship's seawater valves. AS-i (Actuator Sensor Interface) Network consists of three AS-i Master (Gateways), 51 AS-i slaves, installed next to the valves, AS-i Cable and AS-i Power Supply (one for each AS-i Master). AS-i Master and PLC communicate to each other through Profibus Network. AS-i slaves installed in the Network are all of the same type, with four Digital Inputs (DI) and four Digital Outputs (DQ). Digital inputs are connected with proximity sensors in order to "see" the valve's position and Digital outputs are connected with relays in order to activate the actuator of the valves.

As understood, in order to create such a system that is going to be used in case of installed system malfunction, there must be programmed an HMI and a PLC for the automatic control of the valves as well as install new AS-i Master (Gateways) and AS-i Power supply. There is no need to intervene, as it is not necessary, to the AS-i cable and AS-i slaves. In case of a malfunction in a slave, we lose only a small part of the whole system and in case of a malfunction of AS-i cable (difficult to happen), the repair of the cable is very easy.

\section{METHODOLOGY}

The developed methodology, in order to elaborate this paper, was initially an introduction to SCADA systems and the included categories. The introduction to SCADA systems is essential mainly for two reasons. Firstly, to understand how a 
system works and secondly to understand how a Control and Monitoring system is structured. SCADA systems consist of five categories that should be studied. These categories are: Field devices \& signals, Communication Networks, SCADA operations User Workstations, SCADA server Computers and PLC's. All these categories of SCADA systems are illustrated in the picture below (Fig 1).

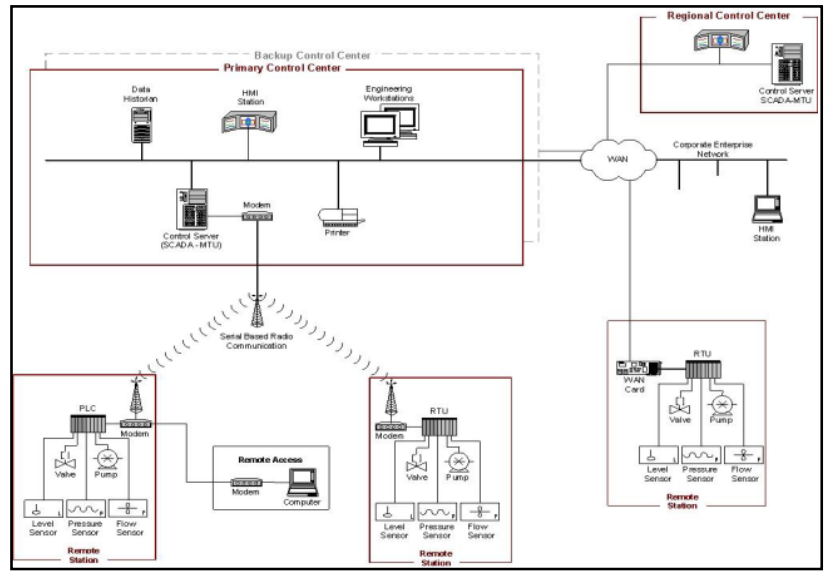

Fig 1: SCADA System General Layout Reference: Guide to Industrial Control Systems (ICS) Security NIST Special Publication 2015 p.2-7

PLC as well as AS-i Network were studied in different chapters. This was done because the system that was going to be made had as key components PLC and AS-i. Therefore, two chapters exist in this thesis about PLC and AS-i Network. In order to understand how a PLC works, a reference was made to the first steps of PLC, how PLC started to replace classic automation boards and the impact they had in the industry. Every company individually has been developing its own controller and networks and there was a mess in the market. It was too difficult to connect PLC from different companies in order to build a Control and Monitoring system. Therefore, industries understood that this mess would not help to improve industry and automation in it. After many efforts and different standards, today there is a standard IEC 61131 that all PLC manufacturers comply with it. Standard IEC61131 in part three, describes the five programming languages divided in two general categories. Every PLC manufacturing industry is obligated to fulfill the minimum requirements of this standard. SIEMENS is one of the biggest industries that produce PLC and complies with standard IEC 61131. PLC S7-1200 is the controller that was used in order to build the backup system. This controller is part of SIEMENS SIMATIC automation components. Therefore, the state of operation, the capabilities of S7-1200, and the hardware that is able to use have been referenced in this chapter.

Last but not least, is AS-i Network. In AS-i Network's chapter, a reference was made to AS-i Bus. AS-i is a fieldbus Network that was developed in 1990. At the same decade, many other fieldbuses were developed but none had the characteristics of AS-i. AS-i is a fieldbus for the lowest levels of the information pyramid in industrial automation technology. It has a wide range of applications in factory automation, process automation, building automation and others. AS-i network was developed in order to connect binary sensors and actuators in the lowest level of automation, which requires very small number of bits to convey device status, through a cable. AS-i transfers Data and energy on a two conductor unshielded cable, named as AS-i cable.
The Architecture of the installed control system is shown in Fig 2. As it was described, the system that controls the ship's valves is part of the Control \& Monitoring system. The system that controls the valves consists of an HMI (Human Machine Interface), a PLC (in which the automation program is executed), AS-i Network and ship's seawater valves. AS-i (Actuator Sensor Interface) Network consists of three AS-i Master (Gateways), 51 AS-i slaves, installed next to the valves, AS-i Cable and AS-i Power Supply (one for each AS-i Master). AS-i Master and PLC communicate to each other through Profibus Network. AS-i slaves installed in the Network are all of the same type, with four Digital Inputs (DI) and four Digital Outputs (DQ). Digital inputs are connected with proximity sensors in order to "see" the valve's position and Digital outputs are connected with relays in order to activate the actuator of the valves. In the scheme, there is shown only one HMI but actually there are more than one, communicating to each other and with the PLC S7-400 via the Industrial Ethernet bus. Communication between HMI and PLC is succeeded through the double Industrial Ethernet, which means that there is redundancy in case of a malfunction of an HMI or in the Ethernet.

As understood, in case of a malfunction in some components of the Control \& Monitoring system, there will be a problem controlling seawater valves. If a malfunction occured in an HMI, there would be no problem because a redundant HMI would be available. In case of a malfunction in the industrial Ethernet, there would be no problem as far as the redundant Ethernet exists. In case of a malfunction in one of the three AS-i Master or in one of the three AS-i power supplies, would mean loss of the entire AS-i line and the slaves. This happens because a redundant system does not exist in case of a failure.

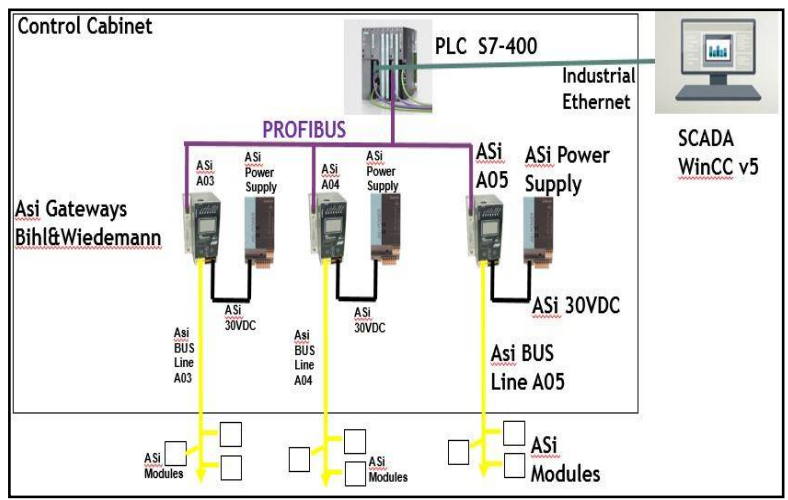

Fig 2: Ship's installed valve control system

In order to fill this gap, a system has been designed, as a backup system in case of a major malfunction. The new (backup) system, should be able to work in case of a failure in one of the components of the existing system such as PLC, the three AS-i Master or AS-i Power supply. To achieve this, a new system should be designed in parallel with the old one. There had to be used three new AS-i Master, three Power supplies (one for each AS-i Line) and one PLC (in order to control AS-i Master) as well as an HMI. From the old system only AS-i lines remained as they were. Two changeover switches were installed per AS-i Line. One for the connection of the new AS-i Master to the Line and one for the connection of AS-i Power supply to AS-i Line. Therefore, in order to switch an AS-i Line from the old system to the new one and control the valves from there, users has to switch changeover switches (AS-i power and AS-i Master) to the new system. As shown in figure 3 , the hardware that was used to implement this system was SIMATIC PLC S7-1214, SIEMENS CM 
1243-2 AS-i Master and SIEMENS AS-i power supply. HMI that was used was also SIEMENS KTP700 Basic.

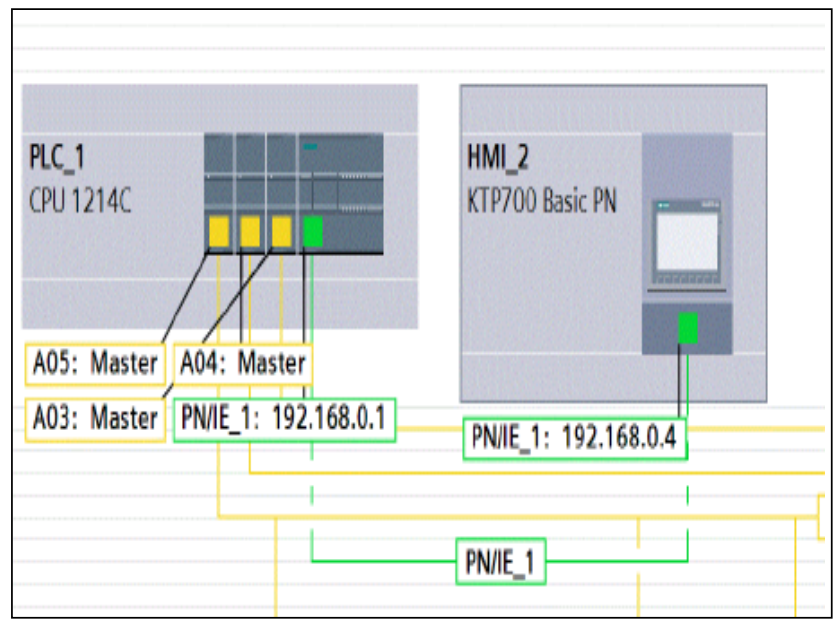

Fig 3: Architecture of the new installed AS-i Master, PLC and HMI

The next step was to program PLC S7-1214C and implement AS-I Master CM1243-2 in the project as well as AS-I slaves for each AS-i Line. There yet had to be searched AS-i addresses of each AS-i slave, and for each proximity as well as Actuators, in order to use these addresses to program PLC, as has happened. Programming PLC S7-1214C and HMI took place according to the users instructions and following the display of the old screen.

\section{CONCLUSIONS}

The biggest problem that had to be clarified was the compatibility of the old AS-i slaves with a new AS-i Master. The technology gap is huge and it was a question if newer AS-i Master could incorporate the old AS-i slaves. Finally this question was answered by AS-i Protocol specifications. Every old AS-i device is compatible with the newer devices. Safety of the Control and Monitoring system has increased according to users and new handling capabilities of the valves have been introduced. A SCADA system should be easily upgradeable to handle future requirements and possible expansions of a system.

\section{ACKNOWLEDGMENTS}

All authors would like to thank the University of West Attica and specifically the Post Graduate Program of Studies (MSc) "New Technologies in Shipping and Transport", for the financial support provided to them to undertake this research project.

\section{REFERENCES}

[1] AS-Interface Organization. (n.d.). retrieved from https://www.as-interface.net/?lang=en

[2] Berger, H. (2016). Automating with SIMATIC (6th ed.). Erlangen: Publicis.

[3] Berger, H. (2013). Automating with SIMATIC S7-1200 (2nd ed.). Erlangen: Publicis.

[4] Berger, H. (2017). Automating with SIMATIC S7-1500 (2nd ed.). Erlangen: Publicis.
[5] Berger., H. (2014). Automating with SIMATIC S7-300 (2nd ed.). Erlangen: Publicis.

[6] Bolton, W. (2015). Programmable Logic Controllers (6th ed.). Waltham MA: ELSEVIER.

[7] Hansen, D. (2015). Programmable Kogic Controllers A practical approach ti IEC 61131-3 Using Codesys. John Wiley \& Sons, Ltd.

[8] IDC Technologies. (2007). Practical Industrial Programming using 61131-3 for PLCs. IDC Technologies.

[9] IDC, T. (2012). Industrial Automation. IDC Technologies.

[10] Kamel, K., \& Kamel, E. (2014). Programmable Logic Controllers Industrial Control. N.Y.: McGraw-Hill Education.

[11] Karl-Heinz, J. (2010). IEC 61131-3: Programming Industrial Automation Systems. N.Y.:Springer.

[12] Krutz, R. (2013). Industrial Automation and Control Principles. ISA.

[13] Lamb, F. (2013). Industrial Automation . Hill.

[14] Manesis, S., \& Nikolakopoulos, G. (2018). Introduction to Industrial Automation. CRC Press.

[15] MCCRADY, S. (2013). Designing SCADA Application Software. London: Elsevier.

[16] Petruzella, F. (2017). Programmable Logic Controllers 5th ed. New York,: McGraw-Hill Education.

[17] Pigan, R. (2008). Automating with PROFINET_Industrial Communication Based on Industrial Ethernet. Erlangen: Publicis.

[18] Reynders, D. (2005). Practical Industrial Data Communications. Elsevier.

[19] SIEMENS. (2013, 04). AS- Interface AS-i Master CM 1243-2 \& AS-i data decoupling unit

[20] DCM 1271. Nurnberg, Germany: SIEMENS.

[21] SIEMENS. (2017, 12). STEP 7 and WinCC Engineering V15 System Manual. Nurnberg, Germany.

[22] SIEMENS. (2018, 08). S7-1200 Programmable controller, System Manual.

[23] Sunit Kumar Sen. (2014). Fieldbus and Networking in Process Automation. CRC Press.

[24] TANENBAUM, A. (1992). Computer Networks. (N. Papandonis, \& K. Karaiskos, Athens: Papasotiriou

[25] Wilamowski, B., \& Irwin, D. (2011). Industrial Communication Systems. CRC Press.

[26] Zurawski, R. (2015). Industrial Communication Technology Handbook. CRC Press.

[27] Marandidis, N. (2000). Automation with SIMATIC S7. Athens: SIEMENS. 